\title{
Frontières
}

ECHARD, B., Souffrance spirituelle du patient en fin de vie : la question du sens, Ramonville-Saint-Agne, Éditions Érès, 2006, $271 \mathrm{p}$.

\section{Andréanne Côté}

Volume 24, numéro 1-2, automne 2011, printemps 2012

URI : https://id.erudit.org/iderudit/1013097ar

DOI : https://doi.org/10.7202/1013097ar

Aller au sommaire du numéro

Éditeur(s)

Université du Québec à Montréal

ISSN

1916-0976 (numérique)

Découvrir la revue

Citer ce compte rendu

Côté, A. (2011). Compte rendu de [ECHARD, B., Souffrance spirituelle du patient en fin de vie : la question du sens, Ramonville-Saint-Agne, Éditions Érès, 2006, 271 p.] Frontières, 24(1-2), 129-130. https://doi.org/10.7202/1013097ar d'utilisation que vous pouvez consulter en ligne.

https://apropos.erudit.org/fr/usagers/politique-dutilisation/ 


\section{ECHARD, B. \\ Souffrance spirituelle du patient en fin de vie: la question du sens}

Ramonville-Saint-Agne, Éditions Érès, 2006, 271 p.

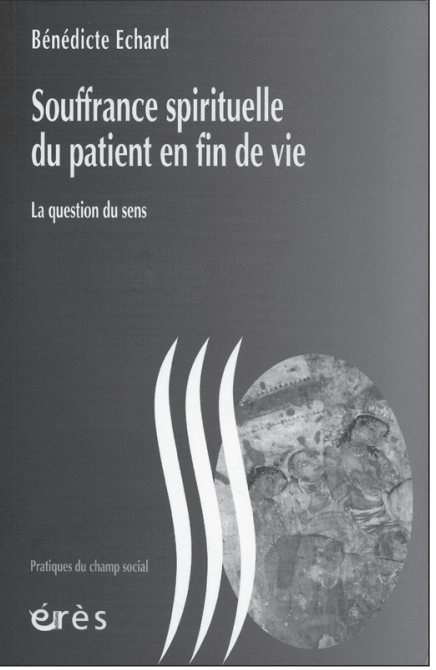

Cet ouvrage, paru il y a cinq ans déjà, n'a pourtant pas perdu en justesse et en pertinence à I'heure des débats sur l'euthanasie et de la Commission spéciale sur la question de mourir dans la dignité. L'auteure, d'origine française, est médecin-homéopathe. Son parcours est également enrichi d'expériences accumulées outremer au sein de l'organisme Médecins sans frontières. Parvenant à allier médecine moderne et thérapies complémentaires, l'ouverture d'esprit qu'elle démontre lui est sans doute utile dans ce domaine encore négligé de la profession médicale que sont les soins palliatifs. Son livre s'adresse d'abord aux aidants naturels et aux intervenants impliqués auprès de personnes atteintes d'une maladie qui menace leur vie à plus ou moins court terme. Parce qu'il jette un regard éclairant sur la question de la spiritualité en fin de vie, il peut aussi bénéficier à quiconque s'intéresse aux avenues thérapeutiques cherchant à intégrer les différents visages de l'être humain, à savoir ses dimensions physique, psychologique, sociale et spirituelle.

À l'ère de la suprématie des sciences biomédicales et de l'évacuation de la religion de l'espace qu'elle occupait jadis, I'homme et la femme doivent maintenant porter seuls le poids de leurs questionnements existentiels à propos de la mort, ce qui n'est pas sans répercussions sur eux. Les besoins spirituels n'en demeurent pas moins présents et essentiels à leur émancipation. S'ils restent inassouvis, ces besoins risquent de se transformer en souffrance spirituelle, notion davantage étudiée, mais encore méconnue des membres de la communauté médicale. II en est surtout question depuis les années cinquante, alors que Cicely Saunders, fondatrice du mouvement des soins palliatifs en Angleterre, travaille à élaborer le concept de douleur totale (Clark 1999). Au fil des histoires partagées au chevet de patient mourants, Dame Saunders constate qu'au-delà d'une douleur physique que l'on parvient à maîtriser, une souffrance spirituelle qui n'est pas prise en compte constitue une atteinte réelle au bien-être et à la qualité de vie. Bien sûr, rendre possible l'émergence d'un sens à partir de l'expérience de la personne souffrante représente un défi de taille, défi que Bénédicte Échard se propose de relever.

Son livre comprend, outre une brève introduction, trois sections qui donnent le ton à sa démarche, humaniste plutôt que théologique: I- Mort, spiritualité et quête de sens, II- Besoins spirituels en fin de vie, III- Questionnement sur le sens, recherche d'un cheminement à travers I'histoire de Marc. Alors que les deux premières parties sont davantage théoriques, la troisième passe par l'histoire d'un homme pour illustrer le propos et poser un regard plus humain sur l'univers de la souffrance spirituelle. La première section propose $d^{\prime} a b o r d ~ u n$ survol historique des conceptions occidentales de la mort à travers le passage du temps. De la mort apprivoisée du Moyen Âge à la mort cachée découlant d'un paternalisme médical, en passant par la mort familière, la mort crainte et la mort impure, le parallèle est intéressant à faire au plan chronologique et idéologique avec le mouvement qui se déploie en route vers la toutepuissance médicale. Culpabilisante envers la science et la médecine, les accusant d'être à l'origine du rêve forcément déçu d'immortalité, Échard n'hésite pas à parler en ces termes en titrant l'une de ses soussections: "La technique, paravent de la mort humaine». Elle en profite par la suite pour camper les définitions des termes qui seront utilisés au cours de l'ouvrage: spiritualité, religion, laïcité, sens et perte de sens, transcendance et sacré.

Dans la deuxième section, l'auteure poursuit l'élaboration de son cadre conceptuel en procédant à l'analyse descriptive des différents besoins spirituels spécifiques à la fin de la vie. Elle les classifie selon quatre groupes: besoins universels, existentiels, de sécurité, d'appartenance et de relation, qui rappellent à quel point l'être humain requiert pour traverser cette épreuve, une présence attentive, empathique, capable d'entendre ce qu'il a à exprimer. À travers le regard de l'autre, les concepts de respect et de dignité prennent tout leur sens. Certes, le fait d'être accompagnée, soignée, entendue, permet de préserver la lumière et l'espoir, mais la personne en fin de vie demeure particulièrement sujette à la dépression et à la démoralisation, menacée par l'intensité de ce moment qui, en vérité, ne peut être que traversé seul.

La troisième section, plus imposante que les précédentes, se divise en trois chapitres: 1) Souffrance et sens, 2) Le sens de la vie, 3) La question du sens de la maladie. Le premier chapitre revisite la théorie de Paul Ricœur sur la souffrance, construite autour des axes soi-autrui et agir-pâtir. II aborde également les représentations symboliques qui se dégagent du récit de la Passion du Christ et du Livre de Job. Ces relectures parlent des dangers de donner un sens et une signification à la souffrance qui sèment la crainte et la peur du jugement, plutôt que l'espoir. L'auteure privilégie davantage un travail de silence, de parole, de présence et de sagesse, nécessaire à I'accompagnement d'une personne en fin de vie. À ce titre, la tradition bouddhiste a beaucoup à nous apprendre. Le second chapitre est déjà plus lumineux, rappelant que le combat contre le mal passe d'abord par le choix de la vie. Ainsi, chaque être est responsable de sa propre quête de sens. Les personnes qui l'entourent, soucieuses de préserver son sentiment d'intégrité et d'unité, ne peuvent que l'assister dans cette démarche personnelle. Redonner la parole au sujet, offrir des soins globaux qui s'adressent à la fois au corps et à l'esprit, sont déjà de bonnes pistes d'intervention. Créer un lien authentique pour favoriser la réconciliation et le pardon est tout aussi indispensable. Le dernier chapitre de l'ouvrage porte spécifiquement sur la question du corps. La conception du corps à travers l'expérience vécue de la maladie, marqué par la dualité corps-esprit, teinte à la fois les attentes et appréhensions du malade, les interventions de la personne qui le soigne, et la relation qui s'établit entre eux. Après avoir habilement résumé différents modèles d'interprétation du corps, l'auteure propose la voie de la phénoménologie pour sortir de l'impasse causée par le modèle du corps organique auquel la médecine anatomo-clinique s'est limitée. Pour Échard, la dimension vivante et subjective du corps sensible rend possible l'émergence du sens au cœur de l'expérience de la maladie.

L'information présentée dans ce livre est riche, et le ton est empreint de compassion à l'égard de ceux qui souffrent. Motivée d'une volonté réelle de soulager et d'aider, Bénédicte Échard parvient à resemer l'espoir en terres ombragées. Toutefois, l'idée maîtresse et les objectifs principaux de son ouvrage auraient mérité d'être clarifiés davantage; une introduction en bonne et due forme permet habituellement d'éviter un tel piège. Renseigner le lecteur dès le départ sur la destination finale rendrait probablement son voyage plus confortable. C'est que la quantité de thèmes abordés et le passage parfois brutal de l'un à l'autre amènent son 
lot de turbulences. Heureusement, la conclusion, présentée de manière claire et concise, propose un bon résumé de la pensée de l'auteure.

La problématique de ce livre est assez centrale dans la réflexion que suscite le développement des soins palliatifs en écho aux avancées technologiques de la médecine curative, sans cesse plus performante dans sa capacité à repousser les limites de la mort. Les points litigieux que Bénédicte Échard soulève en ce qui concerne le paradigme de la science moderne se rapprochent grandement des propos d'autres auteurs avant elle (Toombs, 1992). En sous-estimant la valeur de la réalité subjective d'un sujet souffrant, la médecine moderne annihile son aptitude à faire sens de ce qu'il lui arrive. II en est de même lorsqu'elle réfère aux forces d'auto-guérison immanentes à chacun, forces que la présence délicate de soignants dévoués peut parvenir à raviver (Kearney, 2000). Le regard qu'elle pose sur le corps enrichit la compréhension en faisant place aux avenues thérapeutiques alternatives, telles que les approches psychocorporelles, la méditation, les exercices respiratoires, la relaxation, etc.

En terminant, laissons-nous porter par le passage suivant: "La réflexion sur vie et sens part du principe que c'est le sujet qui donne sens» (Echard, 2006). Cet ouvrage remet à l'honneur le discours au "Je", en redonnant une voix au corps sensible par l'intermédiaire de la phénoménologie. Le sujet qui accepte de se laisser transformer par les épreuves de la vie et de laisser le monde parler à travers lui, se libère d'une partie de sa souffrance à condition qu'une main lui soit tendue. Ce livre touchant rappelle donc la préciosité du lien qui se construit au fil des rencontres et des moments partagés, puisque c'est dans cet espace de silence et de mystère que la guérison intérieure devient possible.

\section{Bibliographie}

CLARK, D. (1999). «'Total pain', disciplinary power and the body in the work of Cicely Saunders, 1958-1967. » Soc. Sci. Med., vol. 49, nº, p. 727-736.

ECHARD, B. (2006). Souffrance spirituelle du patient en fin de vie: la question du sens. Ramonville-Saint-Agne, Éditions Érès.

KEARNEY, M. (2000). A place of healing: working with suffering in living and dying. Oxford, Oxford University Press.

TOOMBS, S. K. (1992). The meaning of illness: the phenomenological account of the different perspectives of physician and patient. Dordrecht, Pays-Bas, Kluwer Academic.

Andréanne Côté

\section{SCHUMACHER, B. N. Quand cesse-t-on de vivre? Pour une définition de la mort humaine}

Nantes, Cécile Defaut, 2011, 119 p.

La technologie moderne a suscité maintes questions bioéthiques et de nombreux débats visant à savoir à quel moment exact un être humain est mort. Si l'on veut être certain de ne pas commettre un meurtre, il est crucial de commencer par définir la

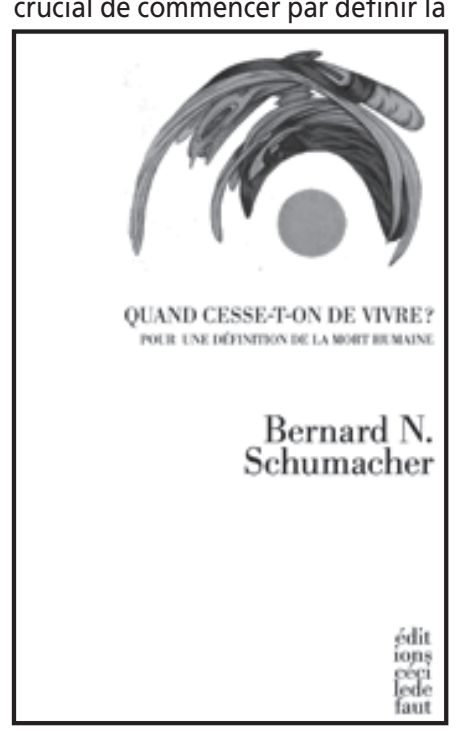

nature de la mort humaine, car une telle définition constitue le point de départ des discussions éthiques sur la fin de vie et l'euthanasie. Dans le présent volume, Bernard N. Schumacher, qui est privatdocent et maître d'enseignement et de recherche en philosophie de I'université de Fribourg, participe à ce débat de manière critique, car il entend montrer les faiblesses des nombreuses définitions de la mort qui sont actuellement proposées par les philosophes.

Parmi les définitions proposées, il y en a une qui retient longuement l'attention de l'auteur: celle de la mort dite néocorticale. Selon Schumacher, cette définition se réfère à une anthropologie qu'il qualifie de dualiste. En effet, les tenants de cette définition supposent que l'organisme humain entretient un rapport extrinsèque avec la personne et que, par conséquent, le corps humain n'est qu'un réceptacle animé de l'esprit. Selon cette logique, la personne humaine est définie uniquement à partir du fonctionnement constaté empiriquement de ses facultés dites personnelles, qu'une analyse comportementaliste est en mesure de confirmer, ou non. Autrement dit, la personne correspond au Moi capable d'exercer ses facultés personnelles, susceptibles d'être repérées de manière empirique. Qui plus est, I'auteur souligne que la définition de la mort dite néocorticale présuppose une anthropologie de l'opérationnalité performante de l'exercice de la conscience de soi, qui légitime la possibilité de traiter, selon certaines modalités d'ordre utilitariste ou conséquentialiste, l'être humain non performant au plan personnel comme une chose dont la valeur serait relative aux intérêts des personnes morales tierces. Schumacher montre également qu'une telle définition de la mort est motivée par des raisons d'ordre principalement pragmatique et utilitariste. Par exemple, il s'agit du plus simple moyen d'atteindre la fin désirable, qui est d'autoriser le prélèvement des organes des enfants anencéphales ou en état de mort néocorticale. En résumé, I'auteur estime que la définition de la mort dite néocorticale conduit à une impasse aussi bien au plan anthropologique qu'au plan éthique. Au plan anthropologique, une telle définition s'enferme dans un subjectivisme auto-fondateur qui conçoit la corporéité comme extérieure au sujet. Au plan éthique, elle conduit à un processus de marginalisation des membres les plus faibles de la communauté humaine de la part de ceux qui auraient sur eux des droits en vertu de leur pouvoir à exercer des facultés dites personnelles.

L'auteur examine ensuite longuement la thèse des philosophes qui proposent de définir la mort en déplaçant le débat du plan anthropologique à celui d'une certaine éthique, notamment une éthique des intérêts. Selon cette thèse, un être humain serait considéré comme mort dans la mesure où l'on aurait évalué, sur la base d'une éthique des intérêts, jointe à un calcul utilitariste et conséquentialiste, qu'une vie humaine placée dans une situation particulière ne vaudrait pas ou plus la peine d'être vécue. Pour les tenants de cette thèse, la définition de la mort s'avère donc logiquement relative à la situation de chaque être humain. Or, I'auteur note que cette proposition repose sur un a priori similaire à celui sur lequel se fonde la définition de la personne émise par les tenants de la mort dite néocorticale, à savoir que, pour être sujet de droits, des droits relatifs à des intérêts, on exige de la personne qu'elle soit en mesure de poursuivre des intérêts, lesquels doivent être empiriquement constatables. Autrement dit, seul celui qui performe des intérêts a des droits. Cette position, précise Schumacher exclut l'existence d'intérêts essentiels à l'être humain, et cela indépendamment de leur exercice, qu'ils soient conscients ou ressentis. Outre cet a priori fortement contestable, Schumacher affirme que c'est une erreur épistémologique de déterminer la mort d'un être humain en choisissant de se situer sur un plan éthique. Un questionnement éthique sur ce qu'il convient ou non de faire d'un être humain se trouvant dans un état particulier ne saurait prétendre répondre à la définition conceptuelle de la mort. Bien au contraire, c'est à partir d'une définition conceptuelle de la mort, qui repose sur une anthropologie philosophique non dualiste que peut se développer une réflexion éthique sur le comportement à adopter dans des situations particulières, notamment les controverses relatives à la transplantation d'organes, au maintien de la vie de manière artificielle et à l'euthanasie.

Pour sortir des impasses des discours qui réduisent la mort soit à sa dimension personnelle sans référence à la dimension corporelle, soit à sa seule dimension biologique sans aucune référence à sa dimension personnelle, l'auteur envisage de re-substantialiser le sujet en désignant la dimension corporelle comme essentielle à la personne humaine. Cette anthropologie, qui considère le corps comme une partie constitutive du sujet, conduit l'auteur à affirmer qu'il y a mort d'une personne lorsqu'il y a destruction de la capacité de l'organisme humain à fonctionner comme un tout et à perpétuer ce processus d'intégrité. L'incapacité de l'une ou l'autre des parties de l'organisme humain ne permet donc pas, à elle seule, de déclarer la mort d'une personne.

Bien entendu, ce bref résumé ne prétend aucunement rendre compte de toute la richesse de cet ouvrage. Par contre, en des temps où tout semble relatif sauf le relativisme, il est certain que cet ouvrage polémique nous propose une vision de l'être humain, de la vie et de la mort plus subtile et nuancée que celle des philosophes qui, au nom d'une éthique séculière, défendent simplement la diversité des définitions de la mort.

Jean-Jacques Lavoie 